\title{
Improvement of numerical instabilities in topology optimization using the SLP method
}

\author{
D. Fujii and N. Kikuchi
}

\begin{abstract}
In this paper, we present a method for preventing numerical instabilities such as checkerboards, mesh-dependencies and local minima occurring in the topology optimization which is formulated by the homogenization design method and in which the SLP method is used as optimizer. In the present method, a function based on the concept of gravity (which we named "the gravity control function") is added to the objective function. The density distribution of the topology is concentrated by maximizing this function, and as a result, checkerboards and intermediate densities are eliminated. Some techniques are introduced in the optimization procedure for preventing the local minima. The validity of the present method is demonstrated by numerical examples of both the short cantilever beam and the MBB beam.
\end{abstract}

Key words Homogenization method, topology optimization, numerical instabilities, sequential linear programming method, two-dimensional structure, finite element method

\section{1}

\section{Introduction}

The topology optimization method for continuum structures has made remarkable progress since the pioneering paper by Bendsøe and Kikuchi (1988), and this method has been applied to various practical problems in the engineering field. Particularly, for the static problem of maximizing the stiffness of structures, it is applied to not only two-dimensional structures (Bendsøe 1995) but also three-dimensional structures (Diaz and Lipton 1997; Olhoff et al. 1998). Recently, it has also been applied to

Received February 23, 1999

D. Fujii and N. Kikuchi

Department of Mechanical Engineering and Applied Mechanics, University of Michigan, Ann Arbor, Michigan 48109-2125, USA

e-mail: dfujii@nasl.t.u-tokyo.ac.jp elasto-plastic problems (Yuge and Kikuchi 1995; Maute et al. 1998). However, it is still not easy to obtain accurate and stable solutions in topology optimization, because a number of problems concerning convergence, checkerboards and mesh-dependencies exist. For the purpose of preventing such numerical instabilities, several methods have been proposed by Haber et al. (1996); Duysinx (1997); Beckers (1997); Petersson and Sigmund (1998), and the current knowledge about these problems summarized by Sigmund and Petersson (1998).

On the other hand, in the topology optimization for continuum structures, the optimality criteria method has been mainly used as an optimizer (Bendsøe and Kikuchi 1988; Suzuki and Kikuchi 1991; Zhou and Rozvany 1991). Particularly, Suzuki and Kikuchi (1991) succeeded in obtaining accurate optimal topology which has a very small mesh-dependence using this method. Recently, as more complicated problems have begun to be dealt with, a mathematical programming method (SLP, SQP) has been used to solve topology optimization problems (Yang and Chuang 1994; Duysinx 1997; Nishiwaki et al. 1998). This is because the mathematical programming method is flexible enough to set any optimization problem. That is, it can theoretically deal with any objective function as compared with the optimality criteria method (Nishiwaki 1998). However, it is not easy to reproduce the optimal topology, which is obtained using the optimality criteria method (e.g. Suzuki and Kikuchi 1991), by using the mathematical programming method. This problem is due to the flatness of the objective function and the numerical optimization procedures of the mathematical programming method.

In this paper, we propose a new method for preventing numerical instabilities occurring when the SLP method is used as the optimizer in the homogenization design method. The proposed method is that a function based on the concept of gravity (which we named the "gravity control function") is added to the objective function. The density distribution of the topology is concentrated by maximizing this function, and as a result, checkerboards and intermediate densities are eliminated. Further, some techniques are introduced in the optimization procedure of the SLP method for preventing local minima. The short cantilever beam is solved as a numerical example 
to demonstrate that the solutions shown by Suzuki and Kikuchi (1991) are nearly reproduced using the present method. The so-called MBB beam (Zhou and Rozvany 1991), which is often adopted to verify the effectiveness of such kinds of methods (Sigmund and Petersson 1998; Haber et al. 1996; Duysinx 1997; Beckers 1997), is also solved as another numerical example to demonstrate the validity of the present method.

The paper is organized as follows. In Sect. 2 we describe the formulation and discretization of the homogenization design method for two-dimensional problems. The topology optimization procedure using the SLP method and the gravity control method is described in Sect. 3 . In Sect. 4 the performance of the gravity control method and some techniques as shown in Sect. 3 are demonstrated using the numerical examples of the short cantilever beam and the MBB beam.

\section{2}

\section{The homogenization design method}

\section{1}

\section{Formulation}

In this section, we give an outline of the homogenization design method proposed by Bendsøe and Kikuchi (1988).

Suppose the periodic microstructures are composed of unit cells with a hole, as shown in Fig. 1. Assume that the displacement field $\mathbf{u}$ can be approximated by an asymptotic expansion

$\mathbf{u}(\mathbf{x}) \approx \mathbf{u}^{H}(\mathbf{x})+\varepsilon \mathbf{u}^{(1)}(\mathbf{x}, \mathbf{y}), \quad \mathbf{y}=\frac{\mathbf{x}}{\varepsilon}$,

where $\mathbf{x}$ is the global macro coordinate, $\mathbf{y}$ is the local micro coordinate, $\varepsilon$ is the representative length of the microstructure, and $\mathbf{u}^{H}, \mathbf{u}^{(1)}$ are expressed for the in-plane problem as follows:

$\mathbf{u}^{H}(\mathbf{x})=\left\{\begin{array}{l}u_{1}^{H} \\ u_{2}^{H}\end{array}\right\}, \quad \mathbf{u}^{(1)}(\mathbf{x}, \mathbf{y})=\left\{\begin{array}{l}u_{1}^{(1)} \\ u_{2}^{(1)}\end{array}\right\}$.

The strain and stress also can be approximated by

$\boldsymbol{\varepsilon}(\mathbf{x}) \approx \varepsilon^{H}(\mathbf{x})+\varepsilon^{(1)}(\mathbf{x}, \mathbf{y}) \approx \mathbf{b}_{\mathbf{x}} \mathbf{u}^{H}(\mathbf{x})+\mathbf{b}_{\mathbf{y}} \mathbf{u}^{(1)}(\mathbf{x}, \mathbf{y})$,

$\boldsymbol{\sigma}(\mathbf{x})=\mathbf{D} \boldsymbol{\varepsilon}(\mathbf{x}) \approx \mathbf{D} \cdot \mathbf{b}_{\mathbf{x}} \mathbf{u}^{H}(\mathbf{x})+\mathbf{D} \cdot \mathbf{b}_{\mathbf{y}} \mathbf{u}^{(1)}(\mathbf{x}, \mathbf{y})$,

where $\mathbf{D}$ is the elasticity matrix, and

$\boldsymbol{\varepsilon}^{H}(\mathbf{x})=\left\{\begin{array}{c}\varepsilon_{1}^{H} \\ \varepsilon_{2}^{H} \\ \gamma_{12}^{H}\end{array}\right\}, \quad \boldsymbol{\varepsilon}^{(1)}(\mathbf{x}, \mathbf{y})=\left\{\begin{array}{c}\varepsilon_{1}^{(1)} \\ \varepsilon_{2}^{(1)} \\ \gamma_{12}^{(1)}\end{array}\right\}$,

$\mathbf{b}_{\mathbf{x}}=\left[\begin{array}{ll}\frac{\partial}{\partial x_{1}} & 0 \\ 0 & \frac{\partial}{\partial x_{2}} \\ \frac{\partial}{\partial x_{2}} & \frac{\partial}{\partial x_{1}}\end{array}\right], \quad \mathbf{b}_{\mathbf{y}}=\left[\begin{array}{ll}\frac{\partial}{\partial y_{1}} & 0 \\ 0 & \frac{\partial}{\partial y_{2}} \\ \frac{\partial}{\partial y_{2}} & \frac{\partial}{\partial y_{1}}\end{array}\right]$.

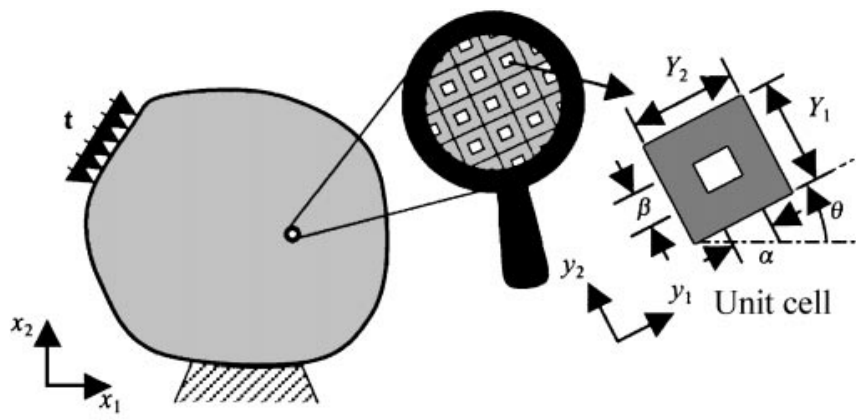

Global macro coordinates Local micro coordinates

Fig. 1 Two-dimensional structure with periodic microstructures

The total potential energy is expanded as

$F(\mathbf{u}) \approx \frac{1}{2} \int_{S}\left(\mathbf{b}_{\mathbf{x}} \mathbf{u}^{H}+\mathbf{b}_{\mathbf{y}} \mathbf{u}^{(1)}\right)^{T} \mathbf{D}\left(\mathbf{b}_{\mathbf{x}} \mathbf{u}^{H}+\right.$

$\left.\mathbf{b}_{\mathbf{y}} \mathbf{u}^{(1)}\right) \mathrm{d} \mathbf{x}-\int_{S}\left(\mathbf{u}^{H}\right)^{T} \mathbf{f} \mathrm{d} \mathbf{x}-\int_{\Gamma}\left(\mathbf{u}^{H}\right)^{T} \mathbf{t} \mathrm{d} \Gamma \approx$

$\frac{1}{2} \int_{S} \frac{1}{|Y|} \int_{Y}\left(\mathbf{b}_{\mathbf{x}} \mathbf{u}^{H}+\mathbf{b}_{\mathbf{y}} \mathbf{u}^{(1)}\right)^{T} \mathbf{D} \times$

$\left(\mathbf{b}_{\mathbf{x}} \mathbf{u}^{H}+\mathbf{b}_{\mathbf{y}} \mathbf{u}^{(1)}\right) \mathrm{d} \mathbf{y} \mathrm{d} \mathbf{x}-$

$\int_{S} \frac{1}{|Y|} \int_{Y}\left(\mathbf{u}^{H}\right)^{T} \mathbf{f} \mathrm{d} \mathbf{y} \mathrm{d} \mathbf{x}-\int_{\Gamma}\left(\mathbf{u}^{H}\right)^{T} \mathbf{t} \mathrm{d} \Gamma$

where $\mathbf{f}$ is the body force vector and $\mathbf{t}$ is the traction force vector. In the last step in (5), we have used the relation

$\lim _{\varepsilon \rightarrow 0} \int_{S} g(\mathbf{x}) \mathrm{d} \mathbf{x}=\int_{S} \frac{1}{|Y|} \int_{Y} g(\mathbf{x}, \mathbf{y}) \mathrm{d} \mathbf{y} \mathrm{d} \mathbf{x}$,

where $g$ is a periodic function in $\mathbf{y}$.

The following equilibrium equations are obtained using the principle of minimum potential energy:

$\frac{1}{|Y|} \int_{S} \int_{Y}\left(\mathbf{b}_{\mathbf{x}} \delta \mathbf{u}^{H}\right)^{T} \mathbf{D}\left(\mathbf{b}_{\mathbf{x}} \mathbf{u}^{H}+\mathbf{b}_{\mathbf{y}} \mathbf{u}^{(1)}\right) \mathrm{d} \mathbf{y} \mathrm{d} \mathbf{x}=$

$\frac{1}{|Y|} \int_{S} \int_{Y}\left(\delta \mathbf{u}^{H}\right)^{T} \mathbf{f} \mathrm{d} \mathbf{y} \mathrm{d} \mathbf{x}+\int_{\Gamma}\left(\delta \mathbf{u}^{H}\right)^{T} \mathbf{t} \mathrm{d} \Gamma$,

$\frac{1}{|Y|} \int_{S} \int_{Y}\left(\mathbf{b}_{\mathbf{y}} \delta \mathbf{u}^{(1)}\right)^{T} \mathbf{D}\left(\mathbf{b}_{\mathbf{x}} \mathbf{u}^{H}+\mathbf{b}_{\mathbf{y}} \mathbf{u}^{(1)}\right) \mathrm{d} \mathbf{y} \mathrm{d} \mathbf{x}=0$. 
If $\mathbf{u}^{(1)}$ is assumed to be decomposed into

$\mathbf{u}^{(1)}(\mathbf{x}, \mathbf{y})=-\chi(\mathbf{y}) \cdot \mathbf{b}_{\mathbf{x}} \mathbf{u}^{H}(\mathbf{x})$,

and if $\chi$ satisfies

$\int_{Y}\left(\mathbf{b}_{\mathbf{y}} \delta \mathbf{u}^{(1)}\right)^{T}\left(\mathbf{D}-\mathbf{D} \cdot \mathbf{b}_{\mathbf{y}} \boldsymbol{\chi}\right) \mathrm{d} \mathbf{y}=0$,

then (8) is automatically satisfied. Substituting (9) into (7) yields the following homogenized equation:

$\int_{S}\left(\mathbf{b}_{\mathbf{x}} \delta \mathbf{u}^{H}\right)^{T} \mathbf{D}^{H}\left(\mathbf{b}_{\mathbf{x}} \mathbf{u}^{H}\right) \mathrm{d} \mathbf{x}=$

$\int_{S}\left(\delta \mathbf{u}^{H}\right)^{T} \mathbf{f}^{H} \mathrm{~d} \mathbf{x}+\int_{\Gamma}\left(\delta \mathbf{u}^{H}\right)^{T} \mathbf{t} \mathrm{d} \Gamma$

where

$\mathbf{D}^{H}=\frac{1}{|Y|} \int_{Y}\left(\mathbf{D}-\mathbf{D} \cdot \mathbf{b}_{\mathbf{y}} \boldsymbol{\chi}\right) \mathrm{d} \mathbf{y}$,

$\mathbf{f}^{H}=\frac{1}{|Y|} \int_{Y} \mathbf{f}(\mathbf{x}, \mathbf{y}) \mathrm{d} \mathbf{y}$

\section{2}

\section{Discretization}

Equation (11) is discretized using the finite element method. In this paper, the nonconforming four-node quadrilateral element proposed by Wilson et al. (1973) and Taylor et al. (1976) is used for the discretization. On the other hand, for microscopic problems the matrix $\chi$ in (12) is obtained by solving (10) under the periodic condition. Equation (10) is also discretized using the fournode isoparametric quadrilateral element (see Fig. 2). The same discretization is applied to the $\mathbf{D}^{H}$ in (12).

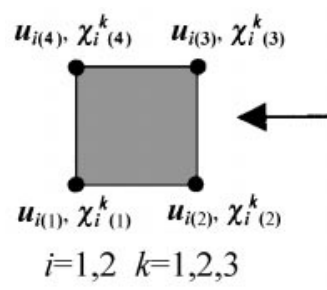

4-node isoparametric finite element

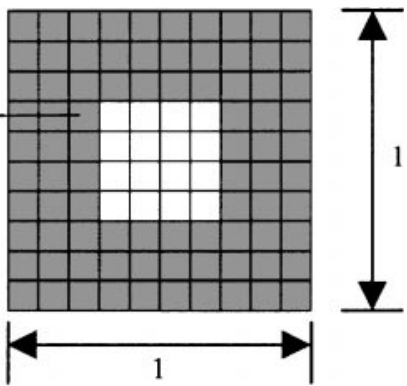

Unit cell

Fig. 2 Discretization of a unit cell

The elasticity matrix $\mathbf{D}^{H}$ for each element of the macrostructure is transformed to the direction of the principal axis of stress by the following equation (Suzuki and Kikuchi 1991):

$\mathbf{D}^{G}=\left(\mathbf{T}^{P}\right)^{T} \mathbf{D}^{H} \mathbf{T}^{P}$,

where

$\mathbf{T}^{P}=\left[\begin{array}{ccc}\cos ^{2} \theta & \sin ^{2} \theta & \cos \theta \sin \theta \\ \sin ^{2} \theta & \cos ^{2} \theta & -\cos \theta \sin \theta \\ -\sin 2 \theta & \sin 2 \theta & \cos 2 \theta\end{array}\right]$.

\section{3}

Optimization procedure using the SLP method

\section{1 \\ Optimization procedure}

The topology optimization problem using the homogenization design method is formulated as follows. Suppose the sizes of a hole of a unit cell are given by $\alpha$ and $\beta$ as shown in Fig. 1, and these sizes are different for each finite element of the macrostructure. The topology optimization consists of finding the optimal sizes $\alpha$ and $\beta$ that minimize the mean compliance of the macrostructure subject to volume constraint of the material. This problem can be formulated as follows:

find $\mathbf{X}=\left\{\alpha_{1}, \alpha_{2}, \ldots, \alpha_{N}, \beta_{1}, \beta_{2}, \ldots, \beta_{N}\right\}$,

which minimize

$C(\mathbf{X})=\int_{S}\left(\mathbf{b}_{\mathbf{x}} \mathbf{u}^{H}\right)^{T} \mathbf{D}^{H}\left(\mathbf{b}_{\mathbf{x}} \mathbf{u}^{H}\right) \mathrm{d} \mathbf{x}$,

subject to

$V(\mathbf{X})=\sum_{i=1}^{N} A_{i} t_{i}\left(1-\alpha_{i} \beta_{i}\right) \leq V_{0}$,

$0 \leq \alpha_{i} \leq 1, \quad 0 \leq \beta_{i} \leq 1 \quad(i=1, \ldots, N)$,

where $N$ denotes the total number of finite elements of the macrostructure, $C$ is the mean compliance, $V$, $V_{0}$ are the total volume of material and the constraint value of $V$, respectively, and $A_{i}, t_{i}$ are the area and the thickness, respectively, of the element $i$.

We use the SLP method to solve the optimization problem defined by (15) and (16). Figure 3 shows the flowchart of the optimization procedure using the SLP method.

The characteristic of this algorithm is that the angles of the principal axis of stress are calculated at the first in each step. This setting is important when the move limits of the design variables are set to large values. 


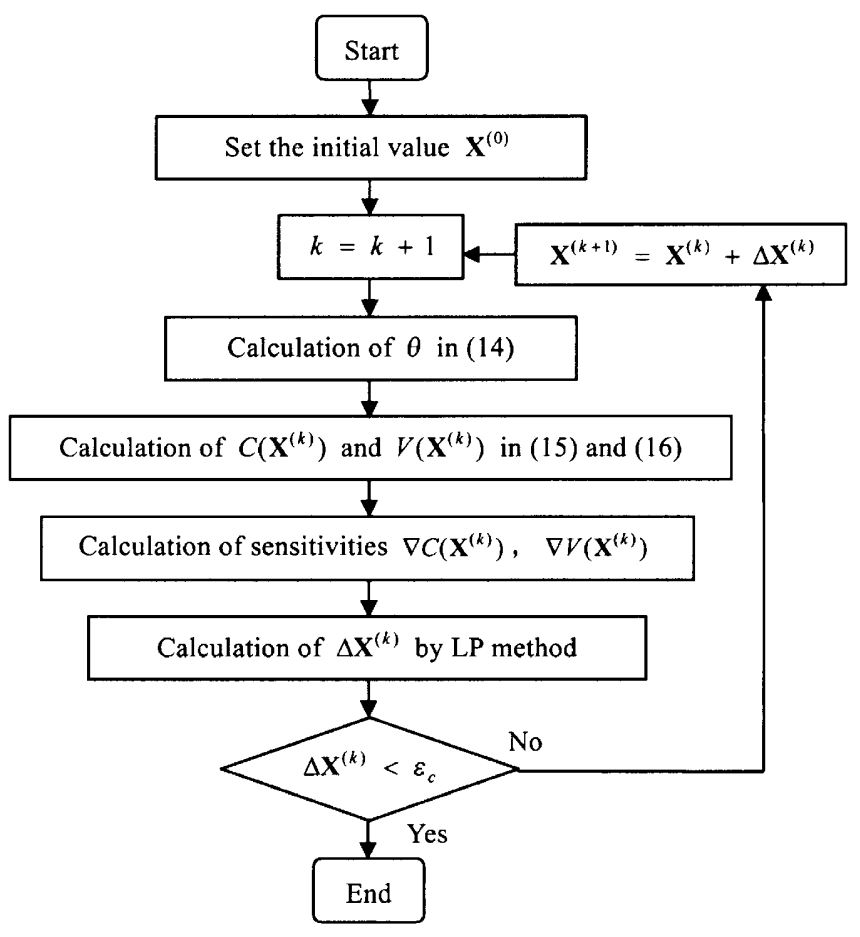

Fig. 3 Flowchart of the optimization procedure

The sensitivities of the mean compliance can be calculated as follows (Suzuki and Kikuchi 1991):

$$
\begin{aligned}
& \frac{\partial C}{\partial \alpha_{i}}=-\int_{S}\left(\mathbf{b}_{\mathbf{x}} \mathbf{u}^{H}\right)^{T} \frac{\partial \mathbf{D}^{H}}{\partial \alpha_{i}}\left(\mathbf{b}_{\mathbf{x}} \mathbf{u}^{H}\right)= \\
& -\mathbf{d}_{i}^{T} \int_{S_{i}} \mathbf{B}_{i}^{T} \frac{\partial \mathbf{D}_{i}^{H}}{\partial \alpha_{i}} \mathbf{B}_{i} \mathrm{~d} \mathbf{x} \cdot \mathbf{d}_{i}, \\
& \frac{\partial C}{\partial \beta_{i}}=-\int_{S}\left(\mathbf{b}_{\mathbf{x}} \mathbf{u}^{H}\right)^{T} \frac{\partial \mathbf{D}^{H}}{\partial \beta_{i}}\left(\mathbf{b}_{\mathbf{x}} \mathbf{u}^{H}\right)= \\
& -\mathbf{d}_{i}^{T} \int_{S_{i}} \mathbf{B}_{i}^{T} \frac{\partial \mathbf{D}_{i}^{H}}{\partial \beta_{i}} \mathbf{B}_{i} \mathrm{~d} \mathbf{x} \cdot \mathbf{d}_{i},
\end{aligned}
$$

where $\mathbf{d}_{i}$ is the nodal displacement vector of element $i$, and $\mathbf{B}_{i}$ the strain-displacement matrix of element $i$. The sensitivities of $\mathbf{D}_{i}^{H}$ are calculated approximately from the data base of $\mathbf{D}^{H}$. In this paper, we made the data base consist of $\mathbf{D}^{H}$ computed at $51 \times 51$ sampling points of $\alpha$ and $\beta$ (the finite element mesh is $20 \times 20$ ). The intermediate values and sensitivities of $\mathbf{D}^{H}$ are calculated by the Lagrange interpolation with 9 points. On the other hand, the sensitivities of $V$ are calculated from (16) analytically.

The design variables are transformed by

$$
\begin{aligned}
& \Delta \tilde{\alpha}_{i}=\frac{\Delta \alpha_{i}}{\alpha_{i}}+\tilde{\varepsilon}, \quad 0 \leq \Delta \tilde{\alpha}_{i} \leq 2 \tilde{\varepsilon}, \\
& \Delta \tilde{\beta}_{i}=\frac{\Delta \beta_{i}}{\beta_{i}}+\tilde{\varepsilon}, \quad 0 \leq \Delta \tilde{\beta}_{i} \leq 2 \tilde{\varepsilon}
\end{aligned}
$$

where $\tilde{\varepsilon}$ usually should be defined $\tilde{\varepsilon}=\varepsilon / \alpha_{i}$ etc., but we deal with $\tilde{\varepsilon}$ as an independent value of $\alpha_{i}$ and $\beta_{i}$. This means the move limit $\varepsilon$ is made proportional to the magnitude of $\alpha_{i}$ and $\beta_{i}$, that is to say, $\varepsilon$ changes in proportion to the magnitude of $\alpha_{i}$ and $\beta_{i}$. In this paper, the initial value of $\tilde{\varepsilon}$ is set to 0.5 , and we change this magnitude by $\tilde{\varepsilon} / 1.05^{n}$ ( $n$ : the iteration number) for $n \leq 30$, and $\tilde{\varepsilon} / 1.05^{n-30}$ for $n>30$. The tolerance $\varepsilon_{c}$ in Fig. 3 is set to $1 / 400$. In this case, the total number of iterations is less than 72 .

Furthermore, the constraint value $V_{0}$ is changed step by step in the following manner when $V_{0}$ is relatively small:

$\tilde{V}_{0}=V_{0}\left(1+\lambda \mu_{n}\right)$,

where $\lambda=V_{T} / V_{0}-2, \mu_{n}=1-(n-1) / n_{s}$, but if $\lambda<0$ or $\mu_{n}<0$, then $\lambda=0, \mu_{n}=0$, and $V_{T}$ denotes the total volume when all $\alpha, \beta$ are equal to zero, and $n_{s}$ is a given number. In this paper, $n_{s}$ is set to 20 . These techniques are effective in preventing the local minima.

\section{2}

\section{Improvement of numerical instabilities}

At present the two major methods, i.e. the perimeter control approach (Haber et al. 1996; Duysinx 1997; Beckers 1997) and the mesh-independent filtering approach (Petersson and Sigmund 1998), have been proposed in order to avoid numerical instabilities in topology optimization (Sigmund and Petersson 1998). However, these approaches are not effective for the homogenization design method as shown in Sect. 4, because they cannot impose a penalty on the gray scale of the densities.

Therefore, we propose a new method based on the concept of gravity. In this method, the gravity control function is defined as follows:

$$
\begin{aligned}
& g=\sum_{i=1}^{N} \sum_{j=1}^{m_{i}}\left(\varrho_{i}^{p} \cdot \varrho_{j}^{p}+\bar{\varrho}_{i}^{p} \cdot \bar{\varrho}_{j}^{p}\right), \\
& \varrho_{i}=1-\alpha_{i} \beta_{i}, \quad \bar{\varrho}_{i}=1-\varrho_{i},
\end{aligned}
$$

where $N$ denotes the total number of the finite elements, $m_{i}$ is the number of elements which have a common side with the element $i\left(m_{i}=4\right.$ for interia elements, $m_{i}=3$ or 2 for edge or corner elements), and $p$ is the penalization factor for the grey scales. In this paper, $p$ is set to 2 . "The gravity control function" is named as such due to the fact that the gravity between two elements is defined by $\varrho_{i} \varrho_{j} / r_{i j}^{2}\left(r_{i j}\right.$ : the distance between two elements).

Figure 4 shows the values of the gravity control function of the element $i$ for the fundamental combinations of elements whose densities are $0,0.5$, and 1 . Figure 5 shows the values of the perimeter control function of the element $i$ in order to compare them with the values in Fig. 4, 


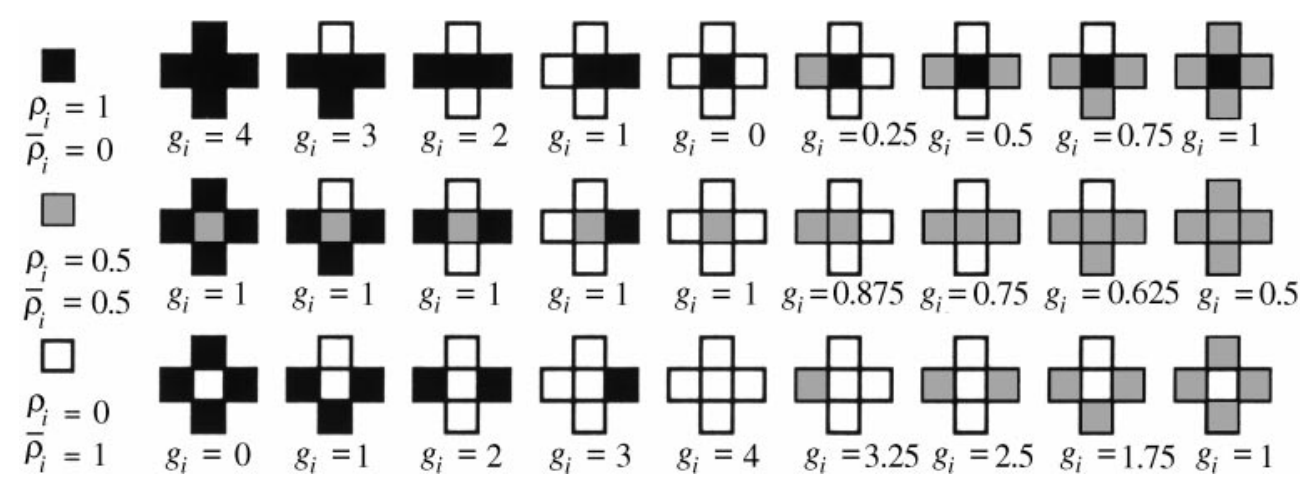

Fig. 4 The values of the gravity control function for the fundamental combinations of elements with densities of $0,0.5$, and 1

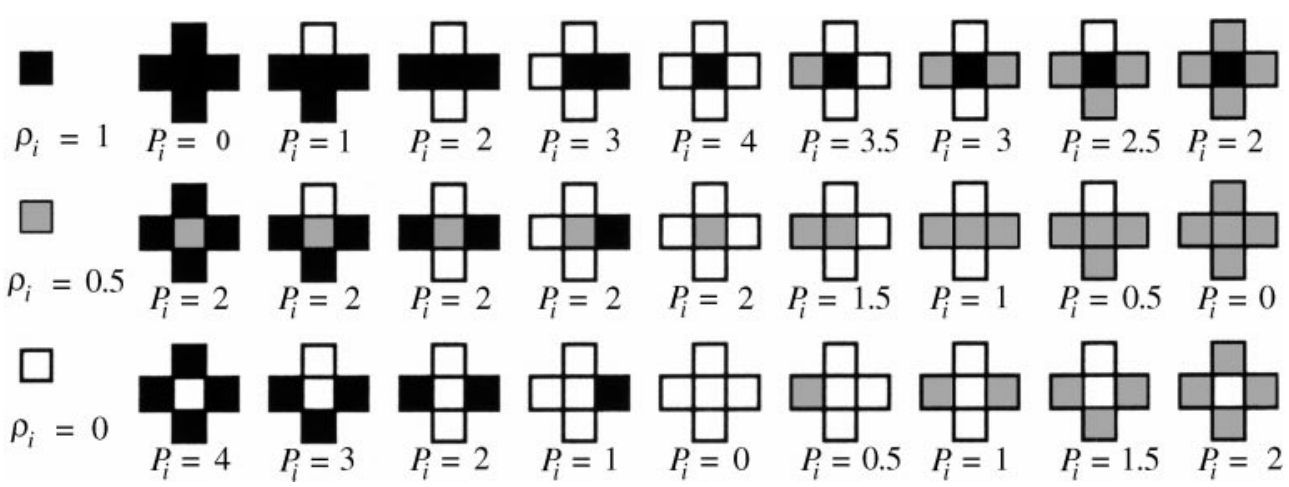

Fig. 5 The values of the perimeter control function for the fundamental combinations of elements with densities of 0 , 0.5 , and 1 $\left(\ell_{i j}=1\right)$

where the perimeter control function is defined by

$P=\sum_{i=1}^{N} \sum_{j=1}^{m_{i}} \ell_{i j}\left|\varrho_{i}-\varrho_{j}\right|$,

and $\ell_{i j}$ is the length of the common side between the $i$ and $j$ elements (Beckers 1997, pp. 108-135). It can be found that the evaluation of both functions is similar when the densities are only 0 or 1 , but in the other case (which density $=0.5$ is included), the values of the gravity control function for gray scales are sufficiently penalized (e.g. 4 for all black and 0.5 for all grey), as compared with the perimeter control function (e.g. 0 for all black and 0 for all grey).

It is clear that as the value of the gravity control function increases, the density of the macrostructure is concentrated and the grey scales are eliminated. Therefore, in this paper, the multiobjective function, in which the mean compliance is minimized and the gravity control function is maximized, is defined as follows:

$$
\begin{aligned}
& f(\mathbf{X})=\sqrt{C(\mathbf{X})^{2}-\left[w_{g} \bar{C} \tilde{g}(\mathbf{X})\right]^{2}}, \\
& \tilde{g}(\mathbf{X})=\frac{g(\mathbf{X})}{\sum_{i=1}^{N} m_{i}},
\end{aligned}
$$

where $w_{g}$ is the weighting ratio between the mean compliance and the gravity control function, $C$ and $\bar{C}$ are the mean compliance $(C=\bar{C}$ in each step), but $\bar{C}$ is independent of the design variables (i.e. $\partial \bar{C} / \partial \mathbf{X}=0$ ), and $\tilde{g}$ is scaled so that it is always less than 1 .

\section{4}

\section{Numerical examples}

\section{1}

\section{Analysis of the short cantilever beam}

First, the short cantilever beam as shown in Fig. 6 (Suzuki and Kikuchi 1991) is analysed to demonstrate the effectiveness of the gravity control method and the present techniques in the optimization procedure. A halfdomain is used for the analysis considering the symmetric condition. The initial values of $\alpha$ and $\beta$ are set to be the same for all finite elements. The thickness of the plate is 1 , and finite element mesh is $64 \times 20$ in the halfdomain.

Figures 8-10 show the solutions obtained using the present algorithm for the variation from 0 to 0.5 of $w_{g}$ in the case of $V_{0}=40,60,80$. In these figures, the magnitude of density for each finite element is expressed by the colour scale as shown in Fig. 7. It can 


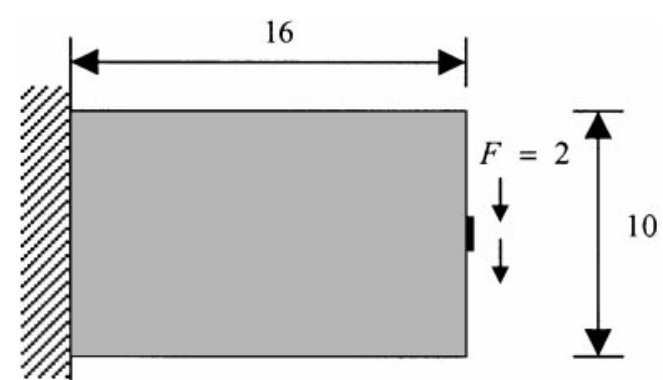

Fig. 6 Design domain of the short cantilever beam

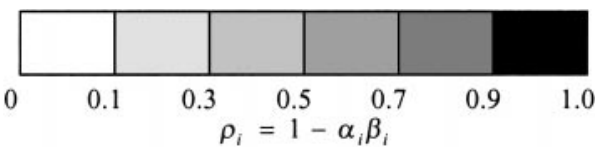

Fig. 7 Colour scale for density variation

be seen that the topologies become clearer as the value of $w_{g}$ increases, and the topologies in the case of $w_{g}=$ 0.3 are similar to the results of Suzuki and Kikuchi (1991).

Figure 11 shows the solutions when the perimeter control function defined by (21) is used instead of the gravity control function. In this case, the multiobjective function is defined by

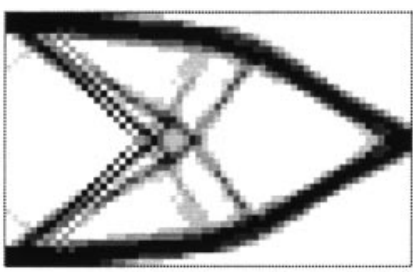

$V_{0}=40, w_{g}=0, C=1.39$

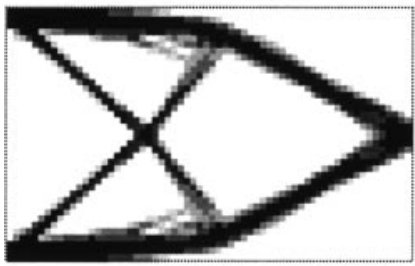

$V_{0}=40, w_{g}=0.2, C=1.47$

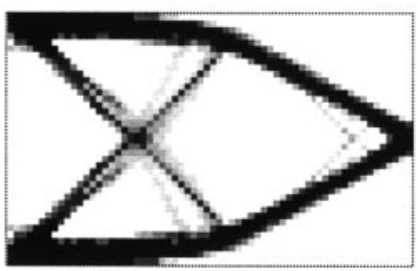

$V_{0}=40, w_{g}=0.4, C=1.53$

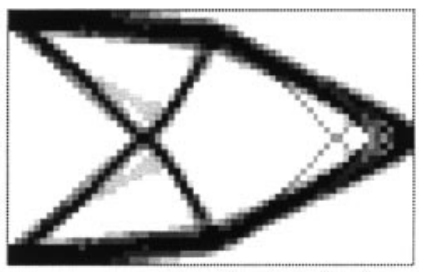

$V_{0}=40, w_{g}=0.1, C=1.42$

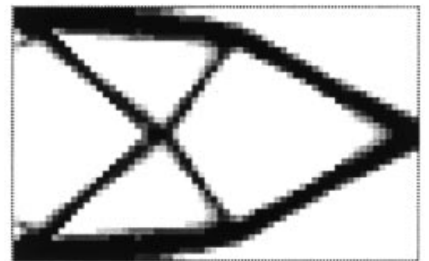

$V_{0}=40, w_{g}=0.3, C=1.49$

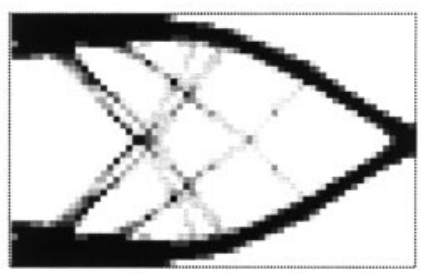

$V_{0}=40, w_{g}=0.5, C=1.72$
Fig. 8 Optimal topologies for the variation from 0 to 0.5 of $w_{g}$ in the case of $V_{0}=40$

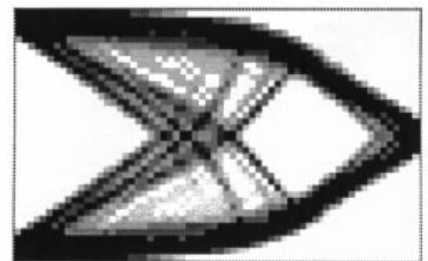

$V_{0}=60, w_{g}=0, C=0.914$

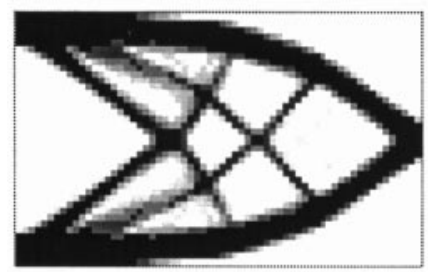

$V_{0}=60, w_{g}=0.2, C=0.925$

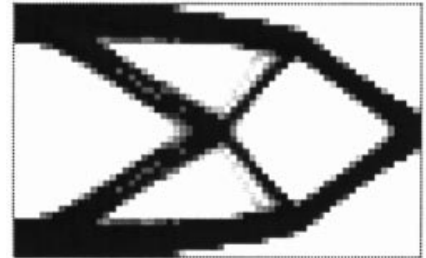

$V_{0}=60, w_{g}=0.4, C=0.986$

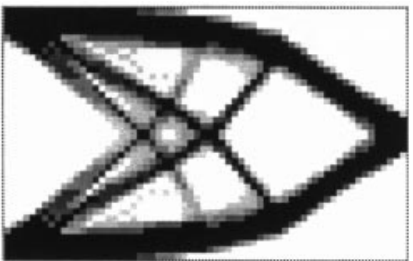

$V_{0}=60, w_{g}=0.1, C=0.922$

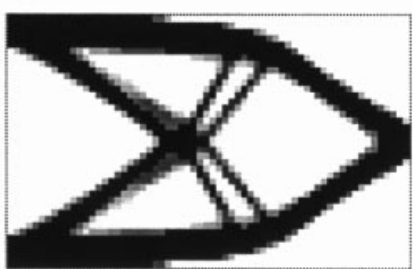

$V_{0}=60, w_{g}=0.3, C=0.943$

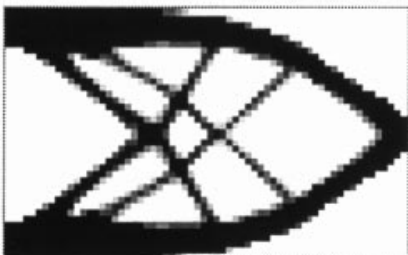

$V_{0}=60, w_{g}=0.5, C=1.05$
Fig. 9 Optimal topologies for the variation from 0 to 0.5 of $w_{g}$ in the case of $V_{0}=60$

$f(\mathbf{X})=\sqrt{C(\mathbf{X})^{2}-\left[w_{p} \bar{C} \tilde{P}(\mathbf{X})\right]^{2}}$,

$\tilde{P}(\mathbf{X})=\frac{P(\mathbf{X})}{L}$,

where we set $w_{p}=0.01$ and $L=16$. It can be seen from these figures that grey scales are not eliminated in this case, and the topology is not clear, especially in the case of $V_{0}=80$. These results suggest that it is necessary to impose a penalty on the grey scales using another method when we use the perimeter control method. The same things can be said for the mesh-independent filtering method, because this method has an effectiveness similar to the perimeter control method.

Figure 12 shows the comparison between the solutions for the different meshes of $48 \times 30$ and $64 \times 40$ in the full domain in order to demonstrate the effectiveness in the mesh dependencies, where $w_{g}=0.3$. It can be seen from these figures that similar topologies can be obtained for the different meshes.

\section{2}

\section{Analysis of the MBB beam}

The so-called MBB beam (Zhou and Rozvany 1991) as shown in Fig. 13 is analysed as the next numerical example. A half-domain is used for the analysis considering 


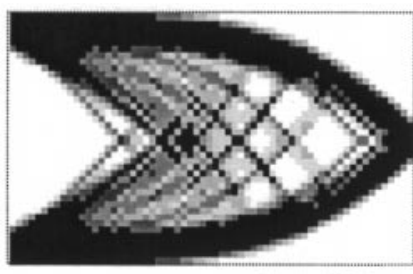

$V_{0}=80, w_{g}=0, C=0.698$

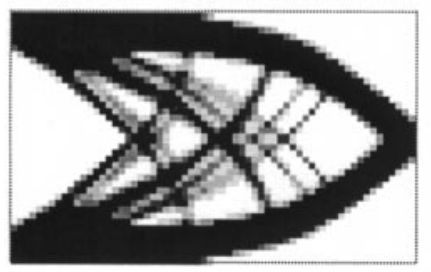

$V_{0}=80, w_{g}=0.2, C=0.708$

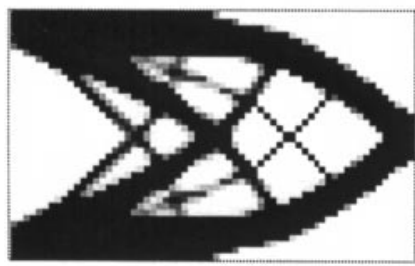

$V_{0}=80, w_{g}=0.4, C=0.738$

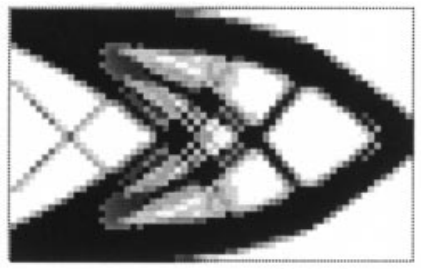

$V_{0}=80, w_{g}=0.1, C=0.711$

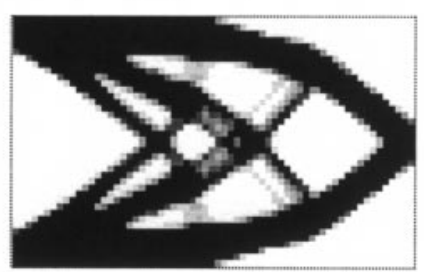

$V_{0}=80, w_{g}=0.3, C=0.717$

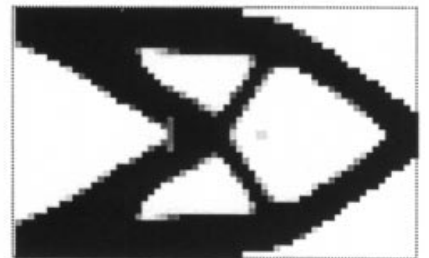

$V_{0}=80, w_{g}=0.5, C=0.787$

Fig. 10 Optimal topologies for the variation from 0 to 0.5 of $w_{g}$ in the case of $V_{0}=80$

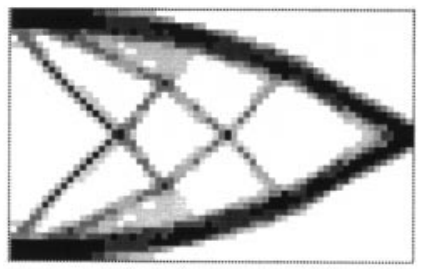

$V_{0}=40, w_{p}=0.01, C=1.41$

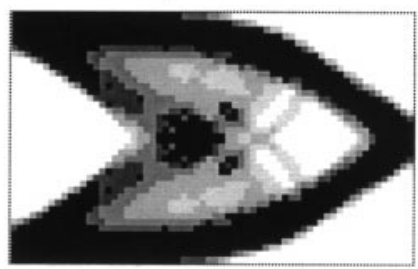

$V_{0}=80, w_{p}=0.01, C=0.704$

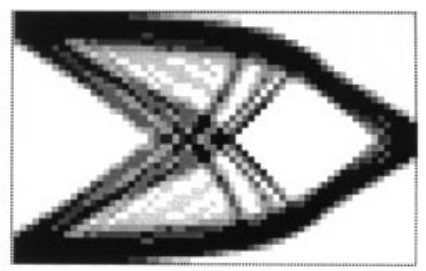

$V_{0}=60, w_{p}=0.01, C=0.914$

Fig. 11 Optimal topologies when the perimeter control function is used instead of the gravity control function

the symmetry. The finite element mesh is $75 \times 25$ in the half-domain. Young's modulus, Poison's ratio, and the thickness of the plate are the same as in the previous example shown in Fig. 6 . $V_{0}$ is set to $50 \%$ of the full density volume $V_{T}$.

Figure 14 shows the solutions obtained using the present algorithm for the variation from 0 to 0.5 of $w_{g}$. It can be found from these figures that like the previous example, the clearest topology is obtained in the case of $w_{g}=0.3$. The topology in the case of $w_{g}=0.3$ is similar to

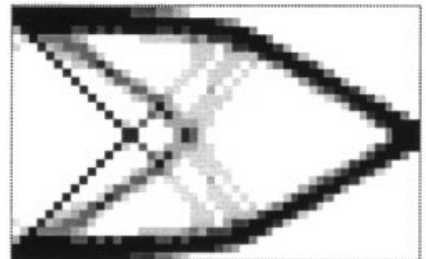

$V_{0}=40$, Mesh $48 \times 30, w_{g}=0.3, C=1.43$

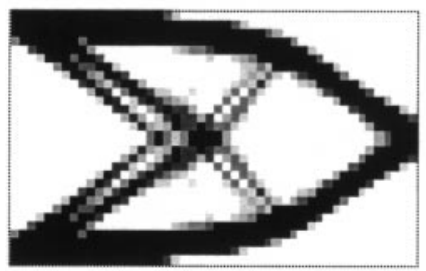

$V_{0}=60$, Mesh $48 \times 30, w_{g}=0.3, C=0.938$

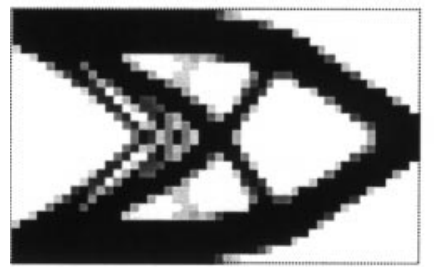

$V_{0}=80$, Mesh $48 \times 30, w_{g}=0.3, C=0.724 V_{0}=80$, Mesh $64 \times 40, w_{g}=0.3, C=0.717$

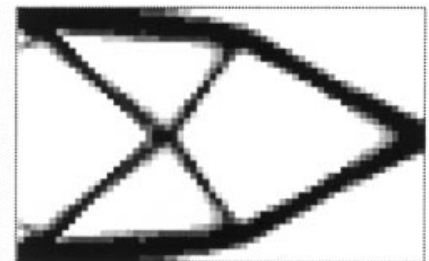

$V_{0}=40$, Mesh $64 \times 40, w_{g}=0.3, C=1.49$

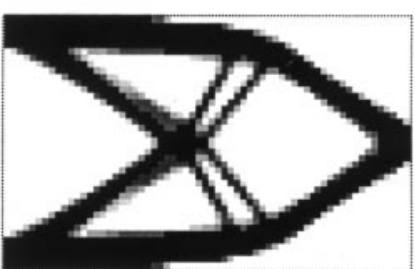

$V_{0}=60$, Mesh $64 \times 40, w_{g}=0.3, C=0.943$

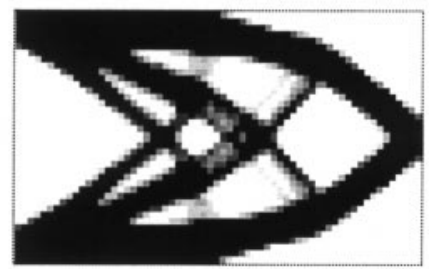

Fig. 12 The comparison between the optimal topologies for the different meshes

the solutions shown by Zhou and Rozvany (1991) which are obtained using the SIMP-DCOC approach. When $w_{g}$ is more than 0.4 , internal lines disappear instead of being concentrated. The final volume is actually $46.5 \%$ in the case of $w_{g}=0.5$. This fact shows that no simpler shape topology solutions exists in this example.

\section{5}

\section{Conclusions}

In this paper, a method is established in order to improve the numerical instabilities occuring when the topology optimization problem is formulated by the homogenization design method and it is solved using the SLP method. The gravity control method, in which the gravity control function is added to the objective function and is then maximized, was presented to eliminate checkerboards and grey scales. A technique, in which the initial value of the move limit of design variables is set to a relatively large value (0.5) and the move limit is made proportional to the magnitude of the design variables, was presented to avoid the local minima. In addition, a technique to relax the volume constraint in the first part of the iteration was presented to avoid the local minima in the case of a relatively small volume constraint (less than $50 \%$ ).

A short cantilever beam was analysed to demonstrate the effectiveness of the present methods, and the results were compared with the solutions presented by Suzuki 


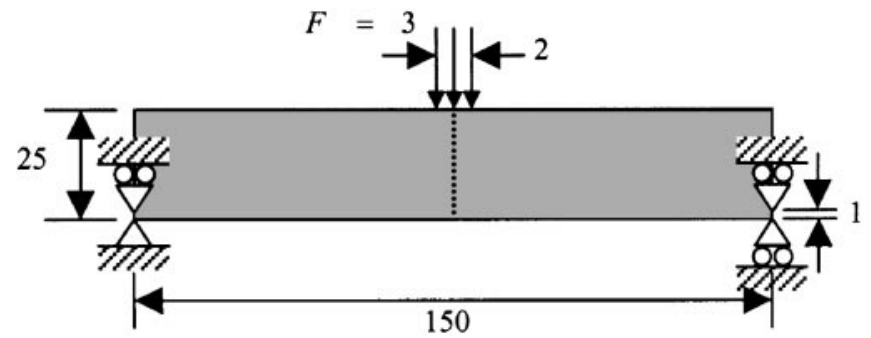

Fig. 13 Design domain of the MBB beam

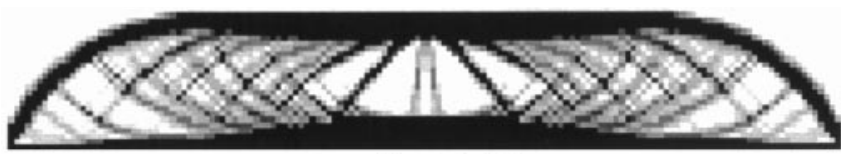

$w_{g}=0, C=3.86$

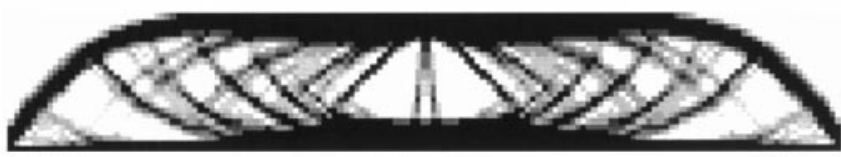

$w_{g}=0.1, C=3.91$

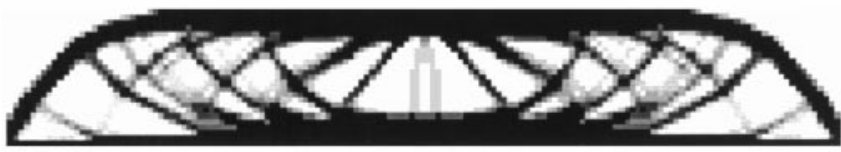

$w_{g}=0.2, C=3.93$

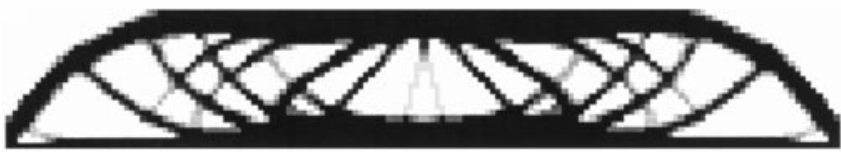

$w_{g}=0.3, C=4.02$

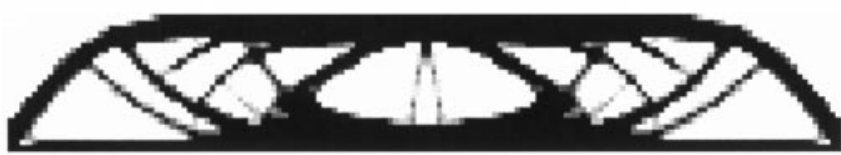

$w_{g}=0.4, C=4.29$

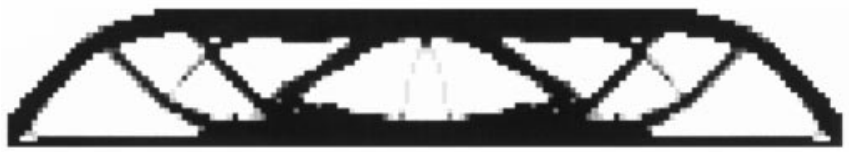

$w_{g}=0.5, C=4.83$

Fig. 14 Optimal topologies of the MBB beam for the variation from 0 to 0.5 of $w g$

and Kikuchi (1991). It could be found from this comparison that the results in which the weighting ratio of the gravity function is set to 0.3 were similar to the solutions obtained by using the optimality criteria method (Suzuki and Kikuchi 1991). The gravity control method was compared with the perimeter control method to demonstrate the characteristics of the present method. It could also be found from this comparison that the grey scales are not penalized by the perimeter control method when compared to the gravity control method. Therefore, the perimeter control method should be combined with a method such as the SIMP approach which has the penalization factor for the grey scales. The solutions for the different finite element meshes were shown to demonstrate the effectiveness in the mesh dependencies. It could be seen from these results that similar topologies can be obtained for the different meshes.

The MBB beam was analysed as the second numerical example. It could be seen from the solutions that the optimal topology in the case of $w_{g}=0.3$ is similar to the solutions shown by Zhou and Rozvany (1991).

It is concluded that if the SLP method is used as the optimizer instead of the optimality criteria method, accurate solutions can be obtained using the method and techniques presented in this paper.

Acknowledgements This work was partially supported by the Nohmura Foundation for Membrane Structures Technology in Japan. Its support is gratefully acknowledged.

\section{References}

Beckers, M. 1997: Optimisation de structures en variables discrètes. Doctoral Thesis, University of Liege

Bendsøe, M.P. 1995: Optimization of structural topology, shape and material. Berlin, Heidelberg, New York: Springer

Bendsøe, M.P.; Kikuchi, N. 1988: Generating optimal topologies in structural design using a homogenization method. Comp. Meth. Appl. Mech. Engrg. 71, 197-224

Diaz, A.; Lipton, R. 1997: Optimal layout for 3D elastic structures. Struct. Optim. 13, 60-64

Duysinx P. 1997: Layout optimization: A mathematical programming approach. DCAMM 540, 1-30

Haber, R.B.; Bendsøe, M.P.; Jog, C. 1996: A new approach to variable topology shape design using a constraint on the perimeter. Struct. Optim. 11, 1-12

Nishiwaki, S. 1998: Optimum structural topology design considering flexibility. Ph. D. Thesis, Mechanical Engineering, The University of Michigan

Nishiwaki, S.; Frecker, M.I.; Min, S.; Kikuchi, N. 1998: Topology optimization of compliant mechanisms using the homogenization method. Int. J. Numer. Meth. Engrg. 42, $535-560$

Maute K.; Schwarz, S.; Ramm, E. 1998: Adaptive topology optimization of elastoplastic structures. Struct. Optim. 15, 81-91

Olhoff, N.; Ronholt, E.; Scheel, J. 1998: Topology optimization of three-dimensional structures using optimum microstructures. Struct. Optim. 16, 1-18

Petersson, J.; Sigmund, O. 1998: Slope constrained topology optimization. Int. J. Numer. Mech. Engng. 41, 1417-1434

Sigmund, O.; Petersson, J. 1998: Numerical instabilities in topology optimization: A survey on procedures dealing with checkerboards, mesh-dependencies and local minima. Struct. Optim. 16, 68-75

Silva, E.C.N.; Fonseca, J.S.O.; Kikuchi, N. 1998: Optimal design of periodic piezocomposites. Comp. Meth. Appl. Mech. Engrg. 159, 49-77 
Suzuki, K.; Kikuchi, N. 1991: A homogenization method for shape and topology optimization. Comp. Meth. Appl. Mech. Engrg. 93, 291-318

Taylor, R.L.; Beresford, P.J.; Wilson, E.L. 1976: A nonconforming element for stress analysis. Int. J. Num. Meth. Eng. 10, 1211-1220

Wilson, E.L.; Taylor, R.L.; Doherty, W.P.; Ghaboussi, T. 1973: Incompatible displacement models. Num. and Comp.
Meth. in Struct. Mech., pp. 43-57 (ed. Fenves, S.T. et al.). Academic Press

Yang, R.J.; Chuang, C.H. 1994: Optimal topology design using linear programming. Comp. \&s Struct. 52, 265-275

Yuge, K.; Kikuchi, N. 1995: Optimization of a frame structure subjected to a plastic deformation. Struct. Optim. 10, 197-208

Zhou, M.; Rozvany, G.I.N. 1991: The COC algorithm, Part II: Topological, geometrical and generalized shape optimization. Comp. Meth. Appl. Mech. Engrg. 89, 309-336 